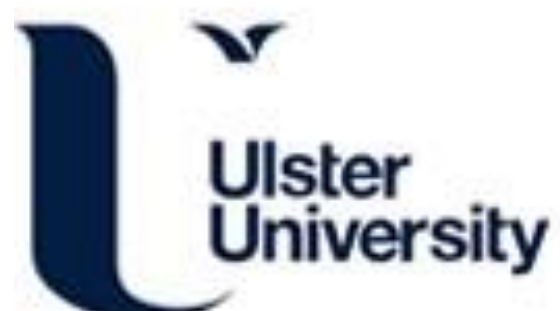

\section{Performance of a Steady-State Visual Evoked Potential and Eye Gaze Hybrid Brain- Computer Interface on Participants With and Without a Brain Injury}

Brennan, C., McCullagh, P., Lightbody, G., Galway, L., McClean, S. I., Stawicki, P., Gembler, F., Volosyak, I., Armstrong, E., \& Thompson, E. (2020). Performance of a Steady-State Visual Evoked Potential and Eye Gaze Hybrid Brain-Computer Interface on Participants With and Without a Brain Injury. IEEE Transactions on HumanMachine Systems, 50(4), 277-286. [9078355]. https://doi.org/10.1109/THMS.2020.2983661

Link to publication record in Ulster University Research Portal

Published in:

IEEE Transactions on Human-Machine Systems

Publication Status:

Published (in print/issue): 01/08/2020

DOI:

10.1109/THMS.2020.2983661

Document Version

Author Accepted version

\section{General rights}

Copyright for the publications made accessible via Ulster University's Research Portal is retained by the author(s) and / or other copyright owners and it is a condition of accessing these publications that users recognise and abide by the legal requirements associated with these rights.

\section{Take down policy}

The Research Portal is Ulster University's institutional repository that provides access to Ulster's research outputs. Every effort has been made to ensure that content in the Research Portal does not infringe any person's rights, or applicable UK laws. If you discover content in the Research Portal that you believe breaches copyright or violates any law, please contact pure-support@ulster.ac.uk. 


\title{
Performance of a Steady State Visual Evoked Potential and Eye Gaze Hybrid Brain-computer interface on Participants with and without a Brain Injury
}

\author{
Chris Brennan, Paul McCullagh, Gaye Lightbody, Leo Galway, Sally McClean,
} Piotr Stawicki, Felix Gembler, Ivan Volosyak, Elaine Armstrong, Eileen Thompson

\begin{abstract}
The brain-computer interface (BCI) and the tracking of eye gaze provide modalities for human-machine communication and control. In this paper we provide the evaluation of a collaborative BCI and eye gaze approach, known as a hybrid BCI. The combined inputs interact with a virtual environment to provide actuation according to a four-way menu system. Two approaches were evaluated: (1) steady state visual evoked potential (SSVEP) BCI with on-screen stimulation; (2) hybrid BCI, which combined eye gaze and SSVEP for navigation and selection. A study comprised participants without known brain injury (non-BI, $\mathrm{N}=30$ ) and participants with known brain injury ( $\mathrm{BI}, \mathrm{N}=14)$. Twenty-nine of the thirty non-BI participants could successfully control the hybrid BCI, while nine out of the fourteen BI participants were able to achieve control, as evidenced by task completion. The hybrid BCI provided a mean accuracy of $\mathbf{9 9 . 8 4 \%}$ in the cohort of non-BI participants and $99.14 \%$ in the cohort of BI participants. Information transfer rates were $\mathbf{2 4 . 4 1}$ bits/min in non-BI participants and $15.87 \mathrm{bits} / \mathrm{min}$ in $\mathrm{BI}$ participants. The research goal is to quantify usage of SSVEP and ET approaches in cohorts of non-BI and BI participants. The hybrid was the preferred interaction modality for most participants for both cohorts. When compared to non-BI participants, it was encouraging that nine out of fourteen participants with known brain injury could use the hBCI technology with equivalent accuracy and efficiency, albeit with slower transfer rates.
\end{abstract}

Index Terms-Brain-computer interface, brain injury, eye tracking, data fusion, virtual environment

\section{INTRODUCTION}

$\mathrm{D}$ isorders that affect the cerebrum and the central nervous system, e.g. stroke, and traumatic brain injury incapacitate the individual concerned, often affecting their mobility and communication. The Future BNCI roadmap estimated stroke prevalence at $3 \%$ of the population, and that 250,000 Americans were living with spinal cord injury (47\% of whom were quadriplegic) [1]. Rehabilitation and long-term care may

This work was submitted for review in March 2019. It was supported by Ulster University. Ethical approval was granted by the Ulster University Research Ethics Committee, REC/16/0053.

Christopher P. Brennan, Paul J. McCullagh, Gaye Lightbody, Leo Galway and Sally McClean are with Computer Science Research Institute, Ulster University, Jordanstown, BT37 0QB, UK (e-mail: brennan-c15@ulster.ac.uk, pj.mccullagh@ulster.ac.uk, g.lightbody@ulster.ac.uk, 1.galway@ulster.ac.uk, si.mcclean@ulster.ac.uk). be required, placing a burden on health and social care systems. In the worst cases, the absence of muscular control means that communication can become difficult or impossible. Eye gaze has been utilised as a human-machine communication channel (e.g. as a computer assisted speller) and is established as an assistive technology for people with impaired peripheral muscle activity but residual eye movement [2]. Eye gaze is measured by an eye tracker (ET); a device which records reflected light from the eye. In particular, ETs have been used to navigate onscreen commands; when a decision or action needs confirmed, 'dwell-time' has been used for activation. However, false positives often ensue [3], and there is a need for a more accurate assistive technology to facilitate communication and control.

The electroencephalogram (EEG) provides a recording of electrical activity emanating from sources within the brain. EEG may be recorded non-invasively by applying electrodes to the scalp and measuring potential differences between each electrode and a reference point. The coupling of the EEG with a computer that can provide processing and actuation is termed a brain-computer interface (BCI). By using exogenous (stimulus-action, e.g. visual stimulation) and endogenous (e.g. motor imagery) paradigms, intentional modulation of brain activity has been used as a mechanism for communication and control. BCI recording has traditionally taken place under controlled laboratory conditions; in more recent years the technology has been made available to users in the community, placing additional emphasis on user requirements of accuracy and ease of use. Indeed, such is the progress and acceptance of this technology that a consumer market has developed led by gaming, mindfulness and wellness applications [4], [5]. BCI provides an alternative user channel to eye gaze, but the approach is less well established as an assistive technology and has usability issues [6].

The steady state visual evoked potential (SSVEP) produces a measurable EEG response to frequency modulated visual stimuli, which can be considered to be representative of a user's interaction with that stimulation frequency [7]. It requires sight

Piotr Stawicki, Felix Gembler, and Ivan Volosyak are with the department of Biomedicine and Engineering, Rhein-Waal University of Applied Sciences, Marie-Curie-Straße 1, 47533 Kleve, Germany (e-mail: piotr.stawicki@hochschule-rhein-waal.de, felix.gembler@hochschule-rheinwaal.de, ivan.volosyak@hochschule-rhein-waal.de. Elaine Armstrong and Eileen Thompson work with The Cedar Foundation (email: E.Armstrong@cedar-foundation.org; E.Thomson@cedar-foundation.org ) 
of and attention to the stimulus. Light sources flickering at frequencies from approximately $8 \mathrm{~Hz}$ to $40 \mathrm{~Hz}$ [8], have been used as the stimuli to elicit responses, which propagate from neuronal sources within the visual cortex. These are most prominent when measured at the occipital region of the scalp. The responses correspond to the fundamental frequencies, as well as higher harmonics, of the eliciting stimuli. EEG-based features that can differentiate the various stimuli can be derived by the use of signal processing techniques [9].

Communication reliant upon EEG features is particularly important for users who have lost peripheral movement (including eye gaze). However, $\mathrm{BCI}$ alone is a difficult assistive technology to establish for a user, as successful deployment requires substantial personalization to the user's needs and individuality within their EEG [10]. Hybrid BCI (hBCI) solutions combining EEG features have the potential to create more effective systems [11], [12]. Solutions that combine ET specifically with BCI have been reported and reviewed in [13], [14]. Typically eye gaze provides robust navigation and BCI paradigms perform a confirmation or switch operation.

In [15] Évain et al combined ET and SSVEP inputs to enhance classification accuracy and demonstrated a speed up in operation and performance over existing gaze hBCI systems, reporting an average speed up of $29 \%$. Stawicki et al [16] demonstrated that greater user control could be achieved by combining SSVEP with ET, with over $90 \%$ of users achieving reliable control of the hBCI, compared to $78 \%$ for eye tracking only and $75 \%$ for pure SSVEP. The motivation in our research was to provide more accurate communication and control for people with brain injury (BI). The research goal is to quantify usage of SSVEP and ET approaches in cohorts of non-BI and BI participant for this purpose. This paper provides the design and evaluation of a collaborative BCI and eye gaze approach, a class of hybrid $\mathrm{BCI}(\mathrm{hBCI})$, which facilitates interaction with virtual environments. The performance of SSVEP was evaluated for both non-BI participants and BI participants, and improvements that $\mathrm{hBCI}$ offers over SSVEP BCI alone were quantified. The assessment of the solution with the BI cohort and its comparison with the non-BI cohort is the major contribution of this work and extends the research in [16]. It is important to stress that this research does not quantify type or extent of brain impairment, but assesses technology use by a group who have previously benefited from assistive technology solutions.

\section{MATERIALS AND METHODS}

\section{A. Collaborative Framework for Interaction}

The hybrid combines input modalities of SSVEP and eye gaze, using the signal processing approach reported in [17], for the BCI actuated component. In previous research (EU-funded BRAIN project, Grant Agreement Number 224156) users with BI provided feedback regarding screen design and user interaction for virtual home communication. Hence this user interface [18] was adopted as the mode of interaction.

The SSVEP paradigm provides an intuitive procedure to collaborate with an ET algorithm. Users interact with an existing menu system [19] using a visual interface application (VIA), which provides navigation of a virtual smart home, as depicted in Fig. 1. The SSVEP paradigm used initially comprised high-frequency stimulation, with external LEDs [18]. However, for ease of navigation the stimuli need to be colocated with the menu options, hence on-screen presentation of the stimuli is more intuitive. Consequently, lower frequency stimuli were adopted as dictated by the display and refresh rates of the liquid crystal display (LCD) monitors. The resultant stimulation rates (typically 6-10 Hz) provide noticeable flicker and hence precludes the use of participants that are photosensitive. However, the signal-to-noise ratio of the evoked activity is higher, thus facilitating feature detection.

Users are required to observe and fixate on the navigation icon they wish to select; the icons are linked to rooms and devices in the environment. For example, from a top-level menu, the living room could be located by first using right or left navigation icons, then selected using the down icon, which would subsequently reveal objects within the living room that could be similarly controlled (e.g. switch a light on/off, change a television channel). As the navigation icons are co-located with frequency-modulated stimuli, the modality for interaction (i.e. eye gaze or SSVEP) does not change from a user interaction perspective. Command selection from the BCI and directional selection from the ET are combined within the data fusion component of the $\mathrm{hBCI}$ system in order to make a collaborative decision. This facilitates control of the VIA using a range of possible input modalities such as EEG alone, eye gaze alone, collaborative EEG and eye gaze, as well as standard input modes (i.e. mouse/keyboard).

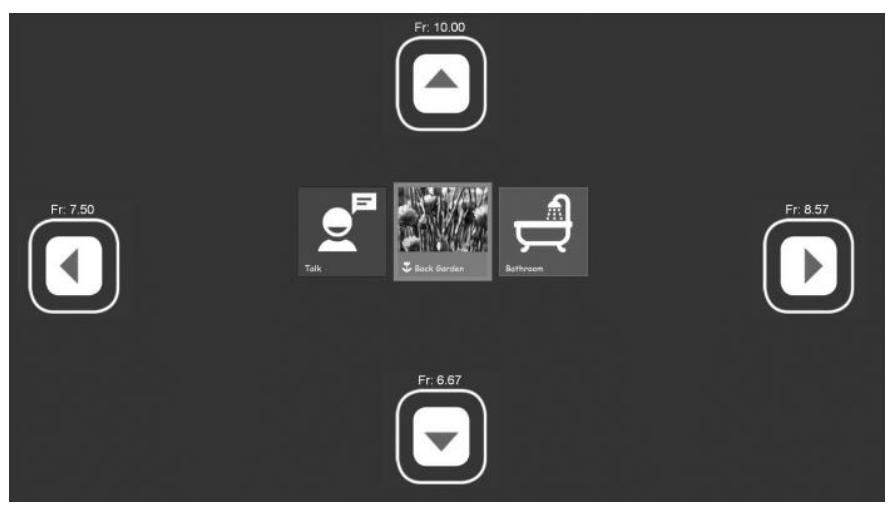

Fig. 1. The Visual Interface Application with on-screen stimuli located at the edges of the display. Each stimulus flickers using unique frequencies (left:7.5 $\mathrm{Hz}$, up: $10 \mathrm{~Hz}$, right:8.57 Hz, down:6.6 7Hz).

\section{B. Recruitment}

This study was undertaken in association with the Cedar Foundation, a charity in Northern Ireland which delivers a range of services that enable people with disabilities to get the most out of life and to be fully included in their communities. Ethical approval was granted by Ulster University Research Ethics Committee (ethics number: REC/16/0053). Participants in the non-BI cohort had no known neurological condition; all participants were excluded from the study if they had photosensitive epilepsy, due to the flashing nature of the stimuli.

Potential end users with a diagnosis of acquired, nonprogressive brain injury (stroke, traumatic brain injury) with differing functional capabilities were considered for 
involvement in the study. BI participants were excluded if they had problems with left-right discrimination, or hearing impairments that could not be corrected with an aid. None of the BI users were dependent on the technology for communication. The number of BI participants was limited by the inclusion criteria and logistical reasons, as recording was limited to a 3-week period. The participant demographics are illustrated in Table I. All participants who removed their spectacles were able to interact with the interface.

\section{Experimental Setup}

TABLE I

RECRUITMENT DEMOGRAPHICS

\begin{tabular}{lll}
\hline \multicolumn{1}{c}{ Metric } & non-Brain Injury & Brain Injury \\
\hline Number & 30 & 14 \\
Recruitment & $\begin{array}{l}\text { Ulster University } \\
\text { staff and students, } \\
\text { public }\end{array}$ & $\begin{array}{l}\text { Cedar service } \\
\text { users }\end{array}$ \\
& No & Token to \\
Remuneration & & cover travel \\
& $21-73$ & $18-67$ \\
Age range (years) & 16 Male; 14 & 12 Male; 2 \\
Gender & Female & Female \\
& $37.6 \pm 14.73$ & $41.6 \pm 13.9$ \\
Age average \pm std dev & 12 & 3 \\
ET experience & 9 & 1 \\
SSVEP experience & 98 & 7 \\
computer experience & 28 & $5(5$ removed \\
Spectacles & $8(6$ removed & glasses $)$ \\
& glasses $)$ &
\end{tabular}

Recordings were conducted at Ulster University (Jordanstown) and in an office setting at the Cedar Foundation (Ballymena). The experimental setup comprised dual LCD displays, an Eye Tribe Tracker as the ET component, a combination of g.USB amplifier, g.LADYbird passive electrodes and a g.GAMMA cap as the BCI component, and a Raspberry Pi home-automation server. Participants were seated approximately $70 \mathrm{~cm}$ from the VIA monitor. The SSVEP paradigm utilised scalp locations $\mathrm{Pz}, \mathrm{PO} 3, \mathrm{PO} 4, \mathrm{Oz}, \mathrm{O} 1, \mathrm{O} 2$, $\mathrm{O} 9, \mathrm{O} 10 \mathrm{AFz}$ (ground) and $\mathrm{Cz}$ (reference) to measure the electrical responses from the visual cortex. Electrode application required a gel-based solution to reduce electrode impedances to approximately $<10 \mathrm{k} \Omega$, measured in the OpenVibe software package [20].

For the non-BI cohort, setup time ranged between 4-26 minutes, average 13:53 minutes ( \pm 5:35 minutes) and total experiment time (two sessions) ranged from 50 minutes to 2 hours and 29 minutes, average 1 hour 25 minutes $( \pm 20: 07$ minutes). For the BI cohort, setup time ranged between 8-39 minutes, average 20:32 minutes $( \pm$ 8:08 minutes) and total experiment time (one session) ranged from 48 minutes to 2 hours and 4 minutes, average 1 hour 24 minutes ( \pm 19:59 minutes). The large time variation was for many reasons; need for experimenter interaction primarily re-calibration, checking electrode impedance and participant breaks which were longer for BI cohort.

\section{Calibration}

For SSVEP calibration, the participant was required to view the flickering stimuli in a sequential manner while the experimenter adjusted thresholds interactively. EEG data were amplified and translated to represent power estimation of the respective stimulation frequency (and harmonics). When this estimation exceeded a predefined threshold, the appropriate directional command was determined. Thresholds varied between 0.5 and 0.3 for each selection (e.g., thresh 1 (left) $=$ 0.45 , thresh $2=0.4$ (right), thresh $3=0.35$ (up), thresh $4=0.5$ (down)). In general, lower stimulating frequencies produced more prominent SSVEP responses, hence individual thresholds were selected corresponding to the frequencies of the stimuli used (and personalised to the participant's EEG). This threshold calibration was subject specific, and depended on the expertise of the experimenter in balancing false positive and false negative command classifications. For participants with a good SSVEP response to a particular stimulating frequency the threshold can be set high. If the response is smaller then the selection may not trigger (i.e., false negative); hence the threshold can be set lower but this risks the selection being triggered by noise in the EEG signal (i.e., a false positive). The four selections must be balanced and this is a subjective variable in the experiment.

For calibration purposes, twenty individual commands (five for each stimulus frequency) were issued by the experimenter to evaluate if the user could successfully execute commands on request. As stated above, the threshold values required for control were different for each participant and adjusted by the experimenter. In general, the flicker of $10 \mathrm{~Hz}$ ('up') tended to produce a lower signal to noise ratio and in most cases this threshold needed to be reduced more than the other frequencies to allow the selection to fire. A sliding time window which provided a dynamic window for power estimation was handled automatically by the SSVEP response detection software and did not required user input as part of the calibration procedure (see Section 11D).

The ET integrates optical sensors, near infra-red signals and image processing components. A source of near infra-red light is reflected from the eyes. A signal acquisition and image processing component recognizes the position of the eyes in real-time and computes the trajectory of user gaze and eye position with respect to a fixation point. The ET device was calibrated prior to a session using nine on-screen gaze points, corresponding to left, middle (horizontal) and right for top, middle (vertical) and bottom of the screen. Accuracy was in the range $\left[0.5^{\circ}-1^{\circ}\right]$. The eye activity was sampled at a rate of $30 \mathrm{~Hz}$, with a latency of $<20 \mathrm{~ms}$. An Eye tribe bespoke algorithm confirmed whether the calibration was deemed appropriate. If not, then re-calibration was required. On-screen coordinates were derived from the trajectory of eye gaze and utilised to determine if gaze location corresponded with one of the onscreen selectable icons.

\section{E. User Interaction}

During sessions, participants were required to follow instructions to complete tasks, controlling the VIA to traverse a hierarchal-menu structure, activating features and functions of a virtual smart home environment. The instructions were issued verbally by the experimenter who remained with the participant, requesting that the participant navigate the menu structure executing four-way control: left, right, up, and down commands. This verbal 'coaching' approach was taken during the first three tasks to ensure that the evaluation was able to 
compare the decisions enacted by the participants, rather than rely on a participant's cognitive ability for menu traversal. Task 1 (domotic control) required participants to interact with lighting in the dining room of the virtual environment. Task 2 (entertainment) required participants to select a television program for playback and subsequently end playback when requested.

In Task 3 (communication) participants were required to navigate to the 'talk' menu and communicate using predefined iconography and computer synthesized speech to indicate 'hunger', i.e. that they wished to have food. In Task 4 (free choice), participants were asked to navigate the interface without instruction to complete a predetermined goal: interact with the kitchen extractor fan and turn it off. For the non-BI cohort Tasks 1- 4 were completed, alternating between SSVEPonly and $\mathrm{hBCI}$ and then in a second session, tasks were completed in the order Task 3, Task 2, Task 1 and Task 4, see Fig 2. The ordering of the tasks within each session was to investigate habituation and learning. Analysis showed that when comparing tasks across session one and session two, t-test $\mathrm{p}$-values were greater than 0.1 for all four tasks. This was for both SSVEP alone and hBCI. This indicated no significant difference across sessions for the non-BI cohort. Hence for the BI cohort only session one was completed, as it was important not to lengthen the recording.

It should be noted that an ET-only condition was not part of this experiment. However, data were collected previously with Tasks 1-3 and these results are reported in section I to serve as a baseline.

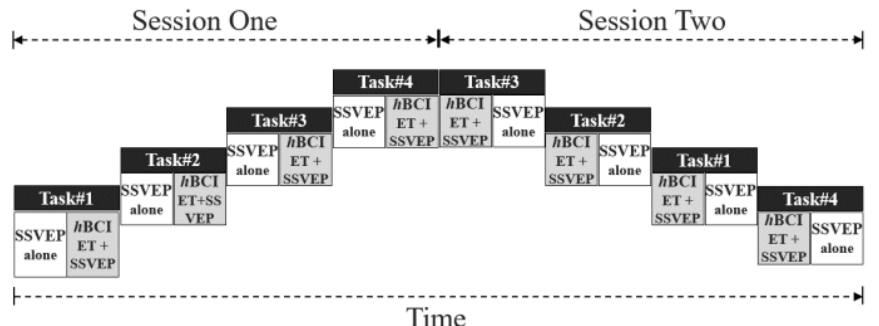

Fig. 2. The structure of the experiment, allowing the collection of data for SSVEP and the $h \mathrm{BCI}$, and accounting for a learning effect or habituation from skewing the data.

If the direction associated with the command selection from the $\mathrm{BCI}$ and the directional selection from the ET were in agreement in the data fusion component, then a collaborative command decision was transmitted to the VIA and the resulting interaction occurred. Where agreement was not obtained, the reason was usually associated with selecting the SSVEP component. The experimenter terminated the session and SSVEP thresholds were altered (as in Section IID) and the session repeated. Where ET was at fault then it was necessary to re-calibrate this component (usually caused by participant movement).

Re-calibration was performed when one of the following conditions was satisfied. For the SSVEP component, (i) a sequence of at least three consecutive incorrect commands were executed and power estimation values were less than $50 \%$ of the threshold for the selection in the quadrant as identified by gaze point; (ii) the frequency detection component identified multiple contradictory frequencies within the same temporal window (window length: $4 \mathrm{~s}$ ) and as a result power estimation could not achieve correct SSVEP threshold. These conditions were indicative of thresholds which were no longer appropriate. For ET, screen-based coordinates were identified within different quadrants within a classification window and as a result gaze-point detection could not achieve the ET threshold. These metrics could be inspected by the experimenter from the software application. The experimenter also used experience and judgement to determine how a session was proceeding. If recalibration was required, the session was repeated from the start. In cases where re-calibration was not successful the session was terminated. Only completed sessions were used in determining the results. The hBCI was conducted after the SSVEP-only experiment and only if a sufficient level of control was achieved using SSVEP-only.

\section{F. SSVEP Response Detection}

Interaction with the VIA uses SSVEP-only or the hBCI. In terms of the BCI component, both SSVEP-only and hBCI modalities apply the same method of on-screen stimulation, electrode positioning, signal acquisition and SSVEP response modelling. As the quality of elicited response to stimulation is crucial for detection of relevant features, it was fundamental that the frequencies of the stimuli were stable and constant. The frequencies utilised were dependent on the refresh rate of the LCD display used and were required to be an exact division of this rate. The on-screen stimuli are located at the edges of the VIA and each stimulus flickers at a different and unique frequency. The refresh rate of the display was set at $60 \mathrm{~Hz}$, and the frequencies selected for stimulation were: $7.5 \mathrm{~Hz}(\mathrm{x} 8=\mathrm{left})$, $8.57 \mathrm{~Hz}$ (x 9= right), $10 \mathrm{~Hz}$ (x 6= up), and $6.67 \mathrm{~Hz}$ (x9 = down).Spatial filtering of the EEG signal was performed using an online adaptation of the Minimum Energy Combination (MEC) method [22] to enhance the EEG information while minimising noise in the signal. The MEC method is applied for extraction of discriminant features. Gembler et al [10] provides a mathematical treatment of the selection criteria for classification of the stimulating frequencies. In our implementation the MEC selects the number of channels required to discard approximately $90 \%$ of the undesirable signals and artefacts. Within a spatially filtered signal, the detection of a produced frequency can be estimated by determining the power $(P)$ in the frequency and a number of harmonics. To avoid overlapping between utilised frequencies and their respective harmonics the number of harmonics was set to 2. If a greater number of harmonics were to be considered, the frequency set would need to be adjusted accordingly. By periodically recalculating the number of channels used, the online adaptation of MEC could utilise those channels that enabled a reduction in energy related to noise by approximately 90\% [21]. Following the process of discriminant feature extraction, power estimation was performed on the spatially filtered signal to facilitate the detection of SSVEP produced frequencies.

Signal classification was then performed based on the probabilities of the frequencies, such that the detected frequency contained the highest probability, had a probability that exceeded a predefined threshold, and represented one of the groups of stimulating frequencies. To enhance signal classification, a sliding window approach was employed for the selection of the time segments of signals used for classification. 
Segment lengths for transferring 8, 20 and 40 blocks of EEG data $(812.5 \mathrm{~ms}, 2031.25 \mathrm{~ms}$ and $4062.5 \mathrm{~ms}$ time epochs respectively) were chosen incrementally for use during SSVEP response modelling on the basis of whether or not classification had already occurred; if no classification could be made, the time segment would be extended up to a maximum of $4062.5 \mathrm{~ms}$ [17].

\section{G. Data Fusion for Collaborative Decisions}

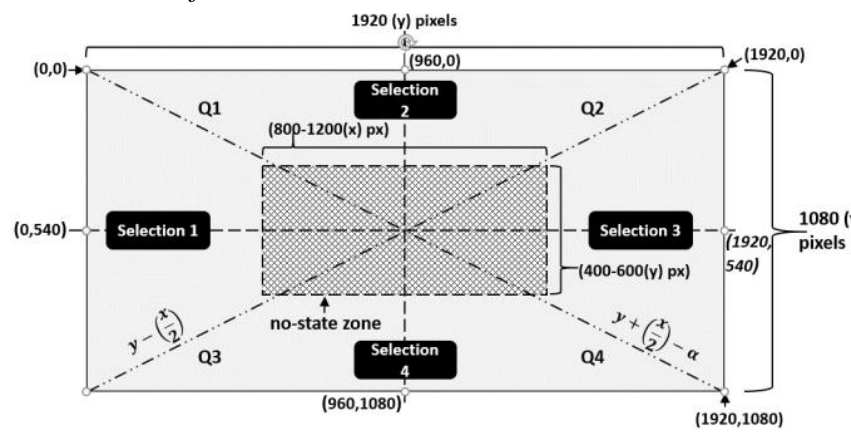

Fig. 3. The theoretical division of a 1920x1080 resolution display into four quadrants, a no-state zone, and the locations of selection icons. $\alpha$ is the horizontal resolution divided by two (i.e. in this case, 960 pixels)

The hBCI utilised an ET in collaboration with the SSVEPbased BCI to issue joint decisions to control the VIA. The ET component undertook acquisition of the user's eye gaze coordinates, which were then passed to the Data Fusion component of the hBCI for further processing. Eye gaze analysis was subsequently performed by dividing the screen into quadrants and designating a central no-state zone, which ensured ET-based directional selection decisions were not made when a user was observing the (changing) menu icons presented on the VIA at the centre of the display, see Fig. 3. To issue commands the user must $d w e l l$ or fixate on the location of one of the selectable icons until the location selection vote reaches a predefined dwell-time threshold, $D_{T}$. The VIA will not trigger menu traversal, however, until the vote for a location selection reaches $D_{T}$ and the BCI agrees, as in the case of the hBCI. In such cases, the Data Fusion component of the hBCI handles this collaborative functionality.

The voting system utilised by the Data Fusion component is based upon the sampling rate of the hardware; the Eye Tribe Tracker samples at $30 \mathrm{~Hz}$, hence generates 30 horizontal $(x)$ and vertical $(y)$ coordinates per second. Therefore, it is possible for 30 votes for a single location selection to occur within one second. By setting a higher $D_{T}$ the minimum time per selection will increase; this reduces the maximum theoretical bit rate. $D_{T}$ was initially set to 50 votes corresponding to a dwell time of 1.6 sec (time spent within the correct quadrant). However, the decision will not be taken until the SSVEP is in agreement. Screen-based coordinates are initially determined using the software development kit (SDK) of the ET. These coordinates allow the voting system to increment or decrement the vote for each location selection and thus enable the Data Fusion component of the hBCI to make decisions that are based upon consistent gaze fixation positions. If the user's fixation point relocates from one quadrant to another, the vote for a location selection will begin to decrement as the vote for an alternative location selection increments. If the user's gaze is off the screen, or in the no-state zone, then voting for all location selections decrements until reaching zero.

\section{H. System Evaluation Metrics}

TABLE II

TASKS FOR SSVEP AND HBCI; SESSION 2 REVERSES THE ORDER, I.E. TASK 3 , TASK 2, TASK1, FOLLOWED BY TASK 4.

\begin{tabular}{|l|l|l|l|l|}
\hline & $\begin{array}{l}\text { Task1 } \\
\text { Domotic } \\
\text { Control }\end{array}$ & $\begin{array}{l}\text { Task2 } \\
\text { Entertainment }\end{array}$ & $\begin{array}{l}\text { Task3 } \\
\text { Commun } \\
\text { ication }\end{array}$ & $\begin{array}{l}\text { Task4 } \\
\text { Kitchen } \\
\text { Fan }\end{array}$ \\
\hline $\begin{array}{l}\text { Number of } \\
\text { commands }\end{array}$ & $\begin{array}{l}13 \\
\text { (Guided) }\end{array}$ & 25 (Guided) & $\begin{array}{l}7 \\
\text { (Guided) }\end{array}$ & $\begin{array}{l}\text { Free } \\
\text { choice }\end{array}$ \\
\hline $\begin{array}{l}\text { Directions } \\
\text { R: Right } \\
\text { L: Left } \\
\begin{array}{l}\text { U: Up } \\
\text { D: Down }\end{array}\end{array}$ & $\begin{array}{l}\text { R-R-R- } \\
\text { R-D-R- } \\
\text { R-D } \\
\text { (Lamp } \\
\text { on)-U-L- }\end{array}$ & $\begin{array}{l}\text { L-L-L-D-L-D- } \\
\text { R-R-D-R-R-- } \\
\text { D-Play)-U-L-L- } \\
\text { D-R-D(Stop)- } \\
\text { L-U-U-R-R-R }\end{array}$ & $\begin{array}{l}\text { L-D-L-L- } \\
\text { D(Eat)- } \\
\text { U-R }\end{array}$ & $\begin{array}{l}\text { More } \\
\text { than one } \\
\text { solution }\end{array}$ \\
& & & \\
\hline
\end{tabular}

In the protocol, participants were guided to complete each task by issuing a series of consecutive directional commands (menu traversal) and selecting the appropriate icon (actuation). Each task was completed after several left, right, up, and down commands were issued in the correct order. Table II shows the optimal number and sequence of commands. Where mistakes occurred, rectifying commands were required. Each task was assessed in terms of Accuracy (Acc), Efficiency (Eff) and Information Transfer Rate (ITR). Acc was calculated as:

$$
\text { Accuracy }=\frac{P_{\text {total }}}{P_{\max }} * 100
$$

where $P_{\text {total }}$ is the total number of correct commands and $P_{\text {max }}$ is the maximum number of commands. However, the value for Acc may provide a misimpression of a participant's performance due to the structure of the tasks; false-positive commands are often succeeded by a command to rectify the mistake, which is defined as an additional correct command. Consequently, the result from participants issuing false-positive commands may suggest enhanced performance, in terms of Acc. For this reason, Eff, as defined by [23], was calculated as:

$$
\text { Efficiency }=\frac{C_{\text {min }}}{C_{\text {total }}} * 100
$$

where $C_{\text {min }}$ is the minimum number of compulsory commands (e.g. 13 for Task 1) and $C_{\text {total }}$ is the total number of detected commands.

ITR in bits per minute (bpm) is the most widely used metric for performance evaluation, defined by [24] and calculated as:

$$
\text { ITR }=\left(\log _{2} M+P \log _{2} P+(1-P) \log _{2}\left[\frac{1-P}{M-1}\right]\right) *\left(\frac{60}{T}\right)
$$

where $M$ is the number of choices, $P$ is the accuracy of target detections, and $T$ (in seconds/selection) is the average time for a selection.

\section{Baseline eyetracking-only evaluation - preliminary work}

A baseline eyetracking-only evaluation was undertaken on 20 participants without any brain injury (ethics number: 2014 0115.14.07). The Eye tribe tracker was used for tasks 1-3, but 
the evaluation was carried out prior to the comparison of SSVEP with the hybrid system. Hence these preliminary results (shown in Fig. 4) can only be used for general comparison.

TABLE III

ACC EFF AND ITR FOR SSVEP-ONLY AND HBCI (NON-BI AND BI COHORTS). N COMPRISES PARTICIPANTS WHO COMPLETED ALL FOUR TASKS.

\begin{tabular}{|c|c|c|c|}
\hline Metric & $\begin{array}{l}\text { non-BI } \\
\text { cohort (mean } \\
\text { and standard } \\
\text { deviation) }\end{array}$ & $\begin{array}{l}\text { BI cohort (mean } \\
\text { and standard } \\
\text { deviation) }\end{array}$ & $\begin{array}{l}\text { Statistical } \\
\text { Comparison between } \\
\text { cohorts }\end{array}$ \\
\hline $\begin{array}{l}\text { SSVEP- } \\
\text { ONLY }\end{array}$ & $\mathrm{N}=29 / 30$ & $\mathrm{~N}=8 / 14$ & \\
\hline $\operatorname{Acc}(\%)$ & $93.30(5.93)$ & $80.26(13.29)$ & $\mathrm{p}<0.01 *(\mathrm{U}$-test $)$ \\
\hline $\operatorname{Eff}(\%)$ & $89.56(9.86)$ & $68.74(19.87)$ & $\mathrm{p}<0.01 *(\mathrm{U}-$ test $)$ \\
\hline ITR(bpm) & $23.78(6.25)$ & $14.23(5.94)$ & $\mathrm{p}<0.01 *(\mathrm{t}$-test $)$ \\
\hline $\mathrm{hBCI}$ & $N=29 / 30$ & $\mathrm{~N}=9 / 14$ & \\
\hline $\operatorname{Acc}(\%)$ & $99.84(0.77)$ & $99.14(1.14)$ & $p>0.10$ \\
\hline Eff $(\%)$ & $99.74(1.23)$ & $99.04(1.18)$ & $\mathrm{p}>0.10$ \\
\hline ITR(bpm) & $24.41(6.35)$ & $15.87(5.66)$ & $\mathrm{p}<0.01 *(\mathrm{t}$-test $)$ \\
\hline
\end{tabular}

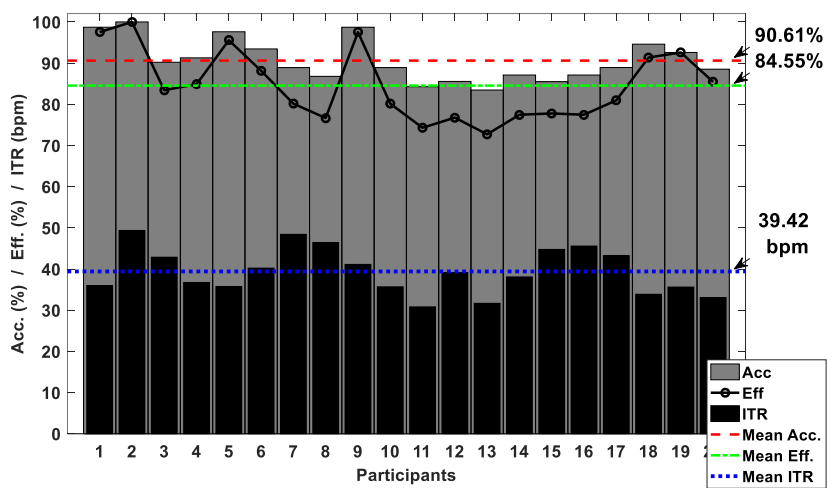

Fig. 4. Non-BI ET (Eye Tribe) evaluation showing a mean Acc. 90.61\%, Eff. $84.55 \%$, and ITR $39.42 \mathrm{bpm}$.

The mean and standard deviation were obtained by averaging over the Task 1, Task 2 and Task 3 for 20 participants. The results indicate a mean Acc of $90.61 \%( \pm 4.96 \%)$, Eff of $84.55 \%( \pm 8.33 \%)$, and ITR of $39.42 \mathrm{bpm}( \pm 5.45 \mathrm{bpm})$. When utilising dwell-time as the selection criterion, some participants were able to achieve over $90 \%$ Acc and Eff, but false-positives occurred when participants were not attempting to interact with the user interface, i.e. at the beginning and end of tasks. Eye tracking control was limited by an inability to prevent the transmission of gaze-based coordinates after intentional control had ended. As an assistive technology, the lack of functionality to start and stop interaction increases the number of falsepositives and reduces the overall performance.

\section{RESULTS}

\section{A. SSVEP-only and hBCI: Non-BI Cohort}

One participant form the non-BI cohort was unable to complete the tasks due to an unreported medical condition. For the remaining twenty-nine, Acc, Eff, and ITR results were obtained from the SSVEP-only and hBCI experiments, see Table III. The ordering of the tasks within each session was to investigate learning as performance may improve for repeated tasks due to experience with the VIA. For session one, Acc was 89\% - 100\% and Eff was 78\% - 100\%. The mean Acc, Eff, and
ITR over all four tasks was $99.71 \%( \pm 1.51 \%), 99.51 \%( \pm$ $2.38 \%)$, and $24.33 \mathrm{bpm}( \pm 7.04 \mathrm{bpm})$. In session two, Acc was 96\%-100\% and Eff 93\%-100\%. The mean Acc, Eff, and ITR over all four tasks was $99.97 \%( \pm 0.17 \%), 99.94 \%( \pm 0.34 \%)$, and $24.49 \mathrm{bpm}$ ( $\pm 6.95 \mathrm{bpm})$, respectively.

Means and standard deviations were obtained by averaging over all participants for individual tasks (session one and two). For SSVEP only, participant Acc was 76\% - 100\% (individual tasks). There was greater variation observed in Eff, with values 59\%-100\% (individual tasks). Metrics were then obtained across all tasks. The mean SSVEP-only Acc, Eff, and ITR for all participants completing the four tasks was $93.30 \%( \pm$ $5.93 \%), 89.56 \%( \pm 9.86 \%)$ and $23.78 \mathrm{bpm}( \pm 6.25 \mathrm{bpm})$, respectively.

For hBCI, the values obtained for Acc varied for the individual tasks, in the range 94\% - 100\% (individual tasks). Likewise, the values obtained for Eff were in the range 89\% $100 \%$ (individual tasks). The overall mean Acc, Eff, and ITR for all participants completing the four tasks was $99.84 \%$ ( \pm $0.77 \%), 99.74 \%( \pm 1.23 \%)$ and $24.41 \mathrm{bpm}( \pm 6.35 \mathrm{bpm})$, respectively. To provide a controlled evaluation, statistical analyses were performed (Acc, Eff and ITR) using SPSS. Shapiro-Wilk tests were conducted to test for normality in the SSVEP-only and hBCI data. This analysis indicated that all metrics in the non-BI cohort collected during the hBCI experiment assume a normal distribution, as do the ITR data from the SSVEP-only experiment. The Acc and Eff data from SSVEP-only, however, are non-normal. The Acc and Eff values are statistically different for the SSVEP-only experiment and the $\mathrm{hBCI}$ experiment, therefore have an unequal variance.

As a further analysis of the two conditions (SSVEP-only and $\mathrm{hBCI}$ ), a non-parametric Wilcoxon signed-rank test indicated that participants performed better using hBCI than with SSVEP-only in terms of Acc p<.001 (z-score $=-4.541)$ and Eff $p<.001(z-s c o r e=-4.541)$. Since the bit rates achieved for both SSVEP-only and hBCI were normally distributed, a paired t-test (parametric) indicated that $\mathrm{hBCI}$ achieved higher ITRs, but without significance ( $\mathrm{p}>.10, \mathrm{z}=-1.643)$.

\section{B. SSVEP-only and hBCI: BI Cohort}

Acc, Eff, and ITR results were collected for SSVEP-only and $\mathrm{hBCI}$ experiments for BI cohort.

For SSVEP-only, participants completed this experiment obtaining Acc in the range 19\% - 100\%, and Eff in the range $0 \%-100 \%$ (individual tasks). Eight participants from the fourteen that took part could successfully complete all four tasks, achieving a mean Acc, Eff, and ITR of $80.26 \%$ ( \pm $13.29 \%), 68.74 \%( \pm 19.87 \%)$, and $14.23 \mathrm{bpm}( \pm 5.94 \mathrm{bpm})$, respectively, see Fig. 5 (a) and Table III. Several participants managed to complete some of the tasks but not all of them. The data indicates that a total of 56 tasks were attempted (4 tasks $x$ 14 participants) and 41 were successfully completed, hence a successful completion rate of $73 \%$.

BI participants who completed the hBCI experiment, obtained Acc and Eff in the range 87\% - 100\% (individual tasks). Nine participants from the 14 that took part could successfully complete all four tasks, achieving a mean Acc, Eff, and ITR of $99.14 \%( \pm 1.14 \%), 99.04 \%( \pm 1.18 \%)$, and 15.87 bpm ( $\pm 5.66 \mathrm{bpm})$, respectively (see Fig. 5 (b)). Several participants managed to complete some of the tasks but not all 
of them. In total, 56 tasks were attempted, 42 were successfully complete, achieving an overall successful completion rate of $75 \%$. In comparison with SSVEP-only one additional task was completed for the cohort: the hBCI was conducted after the SSVEP-only experiment and only if a sufficient level of control, as measured by threshold calibration, was achieved using SSVEP-only.

It is of interest to note that participant 10 provided sufficient threshold calibration to make SSVEP-only selections. These thresholds were not consistent enough to complete any SSVEPonly task. However, when supplemented by ET control the thresholds provided the basis for successful hybrid control. From fourteen BI participants, eight completed all tasks using SSVEP-only and nine completed all tasks using hBCI. From those BI participants that successfully completed all tasks, four failed to achieve 100\% Acc and Eff using hBCI, and yet their performance still increased in contrast with SSVEP-only. Wilcoxon signed-rank test indicated that participants performed better using hBCI than with SSVEP-only. There was a mean Acc increase from $80.26 \%$ to $99.14 \%$ ( $\mathrm{z}$-score $=-2.521$ ), mean Eff improvement of $68.74 \%$ to $99.04 \%$ (z-score $=-2.251$ ), and indeed a mean ITR improvement from $14.23 \mathrm{bpm}$ to $15.87 \mathrm{bpm}$ $(\mathrm{z}$-score $=-1.540)$, Table III.
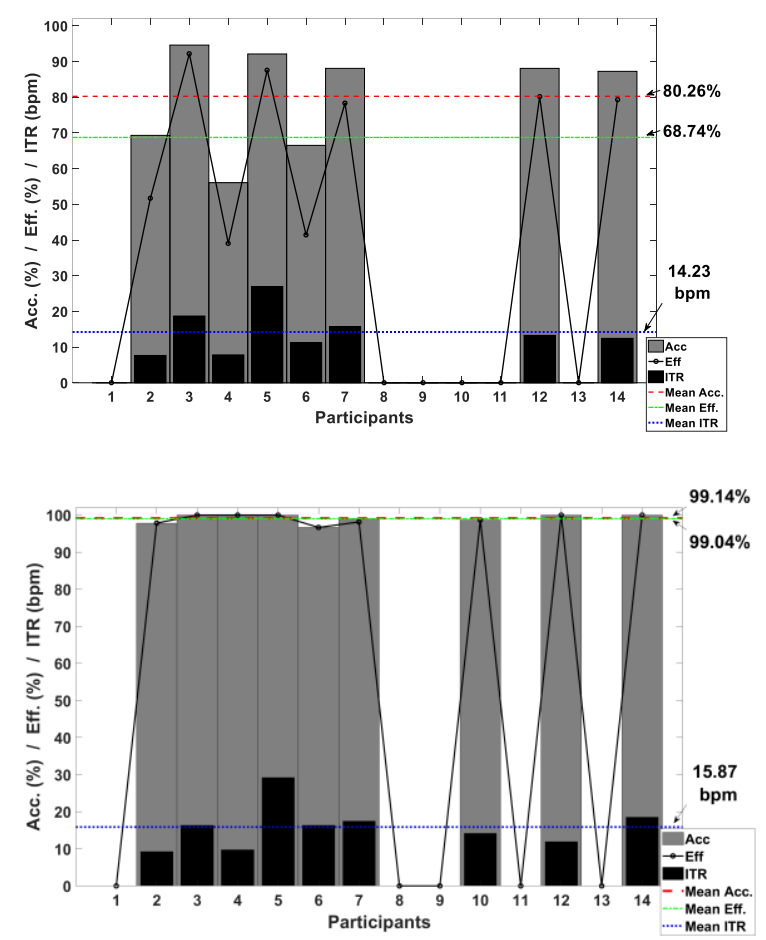

Fig. 5. Data collected from 14 brain-impaired participants: (a) SSVEP-only (mean Acc 80.26\%, Eff 68.74\%\%, ITR 14.23 bpm) (b) hBCI (mean Acc $99.14 \%$, Eff $99.04 \%$, ITR 15.87 bpm).

\section{Comparison of Non-BI and BI Cohorts}

Results indicate that non-BI participants obtained a higher mean Acc, Eff and ITR in comparison to participants from the BI cohort for SSVEP. For hBCI while the ITR was significantly higher for non-BI cohort, differences in Acc and Eff were not significant. The data for non-BI cohort were averaged across two similar tasks (refer to Fig. 2); however, the data for the BCI were for one recorded for one task only (to reduce recording time. Statistical analyses showed no order effect with each comparison between yielding $\mathrm{p}>0.1$.

For both cohorts the performance when contrasted with the results obtained from the use of SSVEP-only (of participants that could successfully use an SSVEP-based BCI) was improved by the hBCI. For example, a participant from the BI cohort who had failed to complete all the tasks with the SSVEPonly, achieved improved performance with the hBCI allowing the completion of all tasks. Through the hBCI, some participants with brain injury may achieve control at an equivalent level as the non-BI cohort, e.g. a participant who had sustained a stroke achieved an average Acc of $92 \%$, Eff of $88 \%$ and ITR of $26.97 \mathrm{bpm}$ using SSVEP-only and an average Acc and Eff of $100 \%$ and an ITR of $29.22 \mathrm{bpm}$ when utilising hBCI.

For SSVEP-only, Shapiro-Wilk tests convey that the data collected during the BI evaluation for all metrics are normally distributed, but the data collected during the non-BI evaluation for Acc and Eff are not normally distributed. A further analysis using the Levene Statistic to check for variance in the data indicated that Acc and Eff had an unequal variance but ITR had an equal variance. It was appropriate to use the Mann-Whitney U-Test to determine any statistical differences in performance between these two cohorts in terms of Acc and Eff. This analysis indicated that non-BI participants performed better than BI participants with a significance of $p<.01$ for Acc, and Eff. As ITR data for both cohorts were normally distributed it was appropriate to use an Independent Samples t-test to determine any statistical difference in performance. This analysis indicates that non-BI participants achieved higher ITR than BI participants with a significance of $p<.01$, which indicates a statistically significant difference between both cohorts. The results of the statistical tests for Acc, Eff and ITR are represented in Table III.

To indicate if there was a statistically significant difference between the performance of non-BI and BI participants when using hBCI, normality tests indicated that Independent Samples t-test should be used for ITR and Mann-Whitney U-Tests for Acc and Eff. This analysis indicated that non-BI participants performed better than BI participants with a significance of $\mathrm{p}<.01$ for ITR. The Mann-Whitney U-Test indicated that nonBI participants achieved higher Acc and Eff scores than BI participants but without significance ( $p>.10)$.

\section{Feedback from participants}

Analysis of post-questionnaire responses from 30 participants in the non-BI cohorts, indicated that five preferred SSVEP-only, 19 preferred hBCI, and six had no preference. Analysis of the post-questionnaire responses from the 14 participants with BI, indicated that two preferred SSVEP-only, eight preferred $\mathrm{hBCI}$, and three had no preference, see Table IV. The remaining participant (C09) was unable to complete any of the tasks. 
TABLE IV

Underlying CONDITION, PERFoRMANCE AND PREFERENCE OF PARTICIPANTS WITH BI

\begin{tabular}{|c|c|c|c|}
\hline$\#$ & Condition & Performance & $\begin{array}{l}\text { Technology } \\
\text { Preference }\end{array}$ \\
\hline $\mathrm{C} 01$ & $\begin{array}{l}\text { traumatic brain } \\
\text { injury }(\mathrm{TBI})\end{array}$ & $\begin{array}{l}\text { Completed Task1.hybrid } \\
\text { and SSVEP BCI and ET } \\
\text { poor }\end{array}$ & No preference \\
\hline $\mathrm{C} 02$ & Stroke & Task1-Task4 & $\mathrm{hBCI}$ \\
\hline $\mathrm{C} 03$ & Stroke & Task1-Task4 & $\mathrm{hBCI}$ \\
\hline $\mathrm{C} 04$ & Cerebral Palsy & Task1-Task4 & $\mathrm{hBCI}$ \\
\hline $\mathrm{C} 05$ & Stroke & Task1-Task4 & SSVEP \\
\hline $\mathrm{C} 06$ & TBI & Task1-Task4 & $\mathrm{hBCI}$ \\
\hline $\mathrm{C} 07$ & TBI & Task1-Task4 & No preference \\
\hline $\mathrm{C} 08$ & TBI & $\begin{array}{l}\text { Completed Task1 and } 3 \\
\text { hybrid and SSVEP. BCI } \\
\text { and ET poor. Difficult to } \\
\text { follow instructions }\end{array}$ & $\mathrm{hBCI}$ \\
\hline $\mathrm{C} 09$ & $\begin{array}{l}\text { Unspecific } \\
\text { nerve disease }\end{array}$ & $\begin{array}{l}\text { SSVEP Signal } \\
\text { processing could not } \\
\text { distinguish frequencies }\end{array}$ & N/A \\
\hline C10 & Stroke & Task1-Task4 & hBCI \\
\hline $\mathrm{C} 11$ & Stroke & $\begin{array}{l}\text { Completed Task1.hybrid } \\
\text { and SSVEP BCI and ET } \\
\text { poor }\end{array}$ & $\mathrm{hBCI}$ \\
\hline $\mathrm{C} 12$ & TBI & Task1-Task4 & $\mathrm{hBCI}$ \\
\hline $\mathrm{C} 13$ & TBI & $\begin{array}{l}\text { Completed Task } 1 \text { and } \\
\text { Task3, participant tired } \\
\text { quickly }\end{array}$ & No preference \\
\hline $\mathrm{C} 14$ & Stroke & Task1-Task4 & SSVEP \\
\hline
\end{tabular}

Six participants $(\mathrm{C} 01, \mathrm{C} 08, \mathrm{C} 09, \mathrm{C} 10, \mathrm{C} 11, \mathrm{C} 13)$ failed to complete all tasks using SSVEP-only. For C10 the hBCI improved performance to allow successful task completion. C01 had sustained a traumatic brain injury (TBI) and titanium plates were fixated under their scalp, which reduced their ability to control the BCI. C08 had also experienced a TBI. This participant's performance was inhibited by their inability to accurately follow instructed commands and their comprehension of the control paradigm, suggesting that poor performance may not be solely attributable to the elicited responses in the brain. This was substantiated by $\mathrm{C} 13$ who elicited a good response to the SSVEP stimuli when engaged in a task but suffered from a high level of fatigue, which prevented the successful completion of all tasks. Their level of performance was equivalent to the highest performing members of the non-BI cohort when engaged, yet they were unable to complete Task 2 and Task 4 due to fatigue. In some cases, alternative BCI paradigms may be more appropriate for users with similar functional deficits who find the flickering stimuli tiring. C09 exhibited a high level of misclassification between the different SSVEP commands. An offline analysis showed no dominant frequency component in the EEG (i.e. the frequency components detected were not evoked by the eliciting stimulus) resulting in misclassification of the SSVEP responses.

\section{DISCUSSION}

The SSVEP paradigm provides a natural and intuitive modality by which to collaborate with an ET algorithm. Participants were required to observe and fixate on a selectable icon. As the set of icons were collocated with visual stimuli, then the technique for interaction did not change from a user perspective. Mean performance metrics of Acc, Eff and ITR improved for the non-BI cohort when comparing hBCI to SSVEP only. Mean performance metrics of Acc, Eff improved for the BI cohort when comparing hBCI to SSVEP only. However, the ITR did not improve for this cohort. The hBCI may contribute to user acceptability as the eye gaze component adds minimal additional user effort to the interaction, while providing inherent reinforcement of the intended selection. Indeed, feedback from both non-BI and BI participants indicated that both cohorts had a general preference for the hBCI.

An analysis of the post-questionnaire responses from the 30 non-BI participants, conveyed that five preferred SSVEP-only, 19 preferred $\mathrm{hBCI}$, and six had no preference. Of the 19 who preferred the hybrid, some stated the hBCI improved confidence during interaction and one user stated 'the hybrid demonstrates a potential for more complex tasks'. Other users substantiated this claim by mentioning that $\mathrm{hBCI}$ seemingly offered enhanced robustness. Two participants contradicted these findings, however, by suggesting that SSVEP was superior as the modality. One participant achieved very good performance using SSVEP-only (Acc 100\%, Eff 100\%, ITR $36.37 \mathrm{bpm}$ ), and $\mathrm{hBCI}$ merely slowed the interaction (Acc $100 \%$, Eff $100 \%$, ITR $33.31 \mathrm{bpm}$ ). Another who also preferred SSVEP-only, achieved a mean Acc of $95.54 \%$, Eff of $93.88 \%$, and ITR of $23.36 \mathrm{bpm}$ utilising SSVEP-only. This participant's qualitative feedback was somewhat surprising considering their performance improved for $\mathrm{hBCI}$ from which they achieved an Acc of $100 \%$, Eff of $100 \%$, and ITR of $21.91 \mathrm{bpm}$ indicating an improvement to both Acc and Eff, albeit with a reduction in ITR. This participant was more tolerant to errors than to an increase in time per selection. One participant who did not achieve 100\% hBCI Acc and Eff found 'the hBCI control restrictive due to the fixed nature of the hardware'. This participant highlights a known restriction of ET technology whereby calibration enforces users to remain stationary; adjusting the seated positioning is known to produce erratic screen-based coordinates.

On a post-recording survey, eight of the participants with BI preferred the SSVEP, two preferred the SSVEP, three had no preference and for one (C09) there was no control achieved. Participants C05 and C14 indicated a preference for SSVEPonly. Both had Acc and Eff of approximately 80\%. C05 had an ITR in excess of 20bpm with SSVEP-only. The hBCI improved the Acc and Eff but there was little change with ITR. It could be argued that this provided sufficient control and the addition of ET was not desired even though it improved the performance. However, this reasoning is not confirmed by C14 where ITR was improved.

The starting point for this research was the use of eye tracking-only. The experience and results obtained from this experiment with 20 participants (non-BI only and a different cohort from that reported in section III) confirm that eye tracking is an intuitive technology that can be used with an ITR of $39.42 \mathrm{bpm}( \pm 5.45 \mathrm{bpm})$, which is higher than $\mathrm{hBCI}$ reported here. However, the mean accuracy of $91 \%( \pm 4.96 \%)$ and efficiency of $85 \%( \pm 8.33 \%)$ indicate that errors still occurred and needed to be corrected in order for the participant to complete the tasks. Users may execute commands in error which would initiate events in the local environment, e.g. windows and doors opening/closing. These data serve as a baseline for the studies reported here using: SSVEP-only and $\mathrm{hBCI}$. The research focus was to determine whether a hybrid 
BCI could provide improved performance through increased accuracy of interaction than BCI alone. However, in retrospect, a third session of eye tracking-only would have been important for comparison and validation.

From thirty non-BI participants, twenty-nine completed the tasks successfully. Of those, only three failed to achieve $100 \%$ Acc and Eff using the hBCI. Mean Acc and Eff, across participants increased by comparison with SSVEP-only. There was a small increasing ITR but no statistically significant difference between these two metrics. In some cases, the ITR for hBCI dropped moderately from SSVEP-only control. These cases represent participants with highly discriminant SSVEP.

By comparison, the performance of the BI cohort was not as good; this was to be expected as a brain injury potentially affects EEG and may also influence concentration and adherence to protocol. Indeed, six of 14 participants from the BI cohort could not use the SSVEP technology. However, for some of the BI cohort, the hBCI performed much better than SSVEP-only. A total of nine participants from the 14 successfully completed all four tasks. This confirms that the subset of the BI cohort performed well with the additional ET technology. For hBCI, ITR is slower than non-BI cohort but the use of collaborative input modalities facilitates increased Acc and Eff to a level comparable to the non-BI cohort. Furthermore, it is interesting to observe that one BI participant could use the hBCI but not the SSVEP-only system.

The Acc metric for the non-BI cohort and the sub-group of the BI cohort (>99\%) for the hBCI compares well with reported accuracy (92.1\%) of a hybrid BCI by Zander in [13]. This study is useful for comparison as eye gaze was employed for navigation and the $\mathrm{BCI}$ component for selection of target search strings located radially around the centre of the screen. The precision of the spatial task (11 distractors and one target) in [13] is more difficult than the 4-way tasks in our experiment. In [15] Évain et al reported that fusion of SSVEP and ET was faster and more accurate than the previous hybrid methods based on sequential processing, where the eye tracking component was responsible for search and BCI component responsible for selection. However, [15] reported that improved speed and accuracy remained lower than interaction based on gaze only. The fusion of classifiers was previously introduced by Muller-Putz et al [25]. In our work we also found enhanced Acc and Eff but lower ITR when compared to a baseline ET study. The work confirms the findings of hBCI in [17]. In their study, the highest ITRs were achieved with ET, but some subjects were not able to gain sufficient control over the standalone ET device or the pure SSVEP system $(78.13 \%$ and $75 \%$ of the participants reached reliable control, respectively). In this respect, their hBCI attained reliable control in $90 \%$ of users and outperformed the pure SSVEP system in terms of user friendliness. The ITR we report for the non-BI cohort is $29 \%$ faster on average.

There are limitations to this research. The SSVEP threshold calibration was session and subject specific, and depended on the expertise of the experimenter in balancing false positive and false negative command classifications. Also, the performance comparison is based on using only one BCI technique, SSVEP detection. Although results cannot be generalized directly to other BCI paradigms and detection/classification methods, the literature ([15], [16]) suggests that this approach has previously been quite successful, a result borne out by our experiments. It would nonetheless be interesting in further work to extend our approach to using different classification methods.

A wizard component that could identify an appropriate subject and stimulus specific threshold could address this situation during calibration, and would add objectivity [10]. Indeed, a fusion approach which combines SSVEP and ET using criteria which are not threshold based would be preferable, requiring less experimenter interaction. Such integration of a wizard component, along with a more robust fusion approach, would be essential to deployment within realworld, home-based scenarios where an experimenter is not available to help maintain the effectiveness of the $\mathrm{hBCI}$ as an assistive technology.

The BI cohort only participated in one session and it is possible that there could be a learning effect which boosted the performance of the $\mathrm{hBCI}$ condition. What is not confirmed from our studies is whether the BI participants who could not use the BCI would have been able to use ET alone at a usable level, albeit with the errors similar to the baseline non-BI cohort. In any further study an ET-only recording session should be introduced to the protocol. Another limitation is that the research does not quantify type or extent of brain injury, but assesses technology use by a group who have benefited from rehabilitation and assistive technology solutions.

With regards to acceptance, the work shows that while nearly all participants without BI could use the technology, only a sub-group of participants with BI could. A much more detailed study would be needed to address this topic, and hence answer the question as to which sub-group of BI participants could benefit from $\mathrm{hBCI}$ as an assistive technology. Expediting the process of identifying potential users would be important. It may be possible to determine at set up whether the SSVEP and ET components are usable by a participant with BI. There is a period of personalization of parameters during the calibration of both ET and SSVEP, which takes about 20 minutes. Hence a person with BI who may go on to use the hBCI technology successfully may be identified quite early in the process. The use of eye tracking does not increase the burden of user interaction significantly and is a useful addition to $\mathrm{BCI}$, as precise eye gaze is not required and hence inexpensive eye tracking hardware can be utilised. It is also possible that some people with BI who may have some control of SSVEP but insufficient control for a BCI-only solution may be able to use the hybrid. However, the evidence for this is sparse, i.e. 8 out of 14 subjects could use the SSVEP and 9 out of 14 could use the $\mathrm{hBCI}$.

Moving BCI technology out of the lab and into the home has long been a goal of BCI research. Requirement B.2.9 of the BNCI Roadmap [1] suggests: "feasibility studies for system durability and especially system performance in home (outside lab) environment". Whilst low cost headsets have become available they do not as yet provide the reliability, durability and accuracy needed. In addition, for any BCI interaction, significant user support is still required with regards to headset application and removal, equipment set-up, calibration and operation. A low cost eye-tracker that contributes to better performance (i.e., enhanced ITR) could aid adoption of BCI as an assistive technology but unconstrained movement may add further calibration issues. As technology, classification 
algorithms and user interfaces improve with possibility of lower cost solutions, the move to home may be possible, initially for non-safety critical applications.

\section{CONCLUSION}

This research indicates that a hybrid solution combining ET with SSVEP BCI can enhance the performance when compared to BCI alone. The SSVEP limits the ITR but interaction is less prone to errors. When comparing participants from non-BI and BI cohorts the accuracy, efficiency and ITR are better in the former cohort for SSVEP-only. However, nine out of fourteen participants from the BI cohort could use the hBCI. Accuracy and efficiency are higher for the non-BI cohort in comparison with the BI participants, but this does not reach significance (non-BI Acc is $99.84 \%$, BI Acc is $99.14 \%, \quad \mathrm{p}>0.10$; non-BI Eff is $99.74 \%$, BI Eff is $99.04 \%, p>0.10$ ). The ITR measure by comparison does reach significance (non BI ITR is $24.41 \mathrm{bpm}$, BI ITR is $15.87 \mathrm{bpm}, \mathrm{p}<0.01$ ). This means that the subset of BI participants was able to use the technology with high accuracy and efficiency; albeit that interaction time was longer. Hence the hBCI improved Acc and Eff performance for the non-BI cohort who can use the system.

\section{REFERENCES}

[1] BNCI Horizon 2020, "Roadmap: The Future in Brain/NeuralComputer Interaction: Horizon 2020," 2015.

[2] C. Vidaurre, A. Kübler, M. Tangermann, K.-R. Müller, and J. del R. Millán, "Brain-computer interfaces and Visual Activity," in Gaze Interaction and Applications of Eye Tracking, IGI Global, pp. 153-174, 2011.

[3] R. J. K. Jacob, "The use of eye movements in human-computer interaction techniques: what you look at is what you get," $A C M$ Trans. Inf. Syst., vol. 9, no. 3, pp. 152-169, 1991.

[4] C. Sas and R. Chopra, "MeditAid: a wearable adaptive neurofeedback-based system for training mindfulness state," Pers Ubiquit Comput, vol. 19, no. 7, pp. 1169-1182, Oct. 2015.

[5] H. Gurkok, A. Nijholt, and M. Poel, "Brain-computer interface Games: Towards a Framework," in Handbook of Digital Games and Entertainment Technologies, chapter 5, Springer Singapore, pp. 133-150, 2017.

[6] F. Nijboer, "Technology transfer of brain-computer interfaces as assistive technology: Barriers and opportunities," Annals of Physical and Rehabilitation Medicine, vol. 58, no. 1, pp. 35-38, Feb. 2015.

[7] B. Allison, D. J. McFarland, G. Schalk, S. D. Zheng, M. M. Jackson, and J. R. Wolpaw, "Towards an independent braincomputer interface using steady state visual evoked potentials," Clinical Neurophysiology, vol. 119, no. 2, pp. 399-408, Feb. 2008.

[8] G. Garcia-Molina and V. Mihajlovic, "Spatial filters to detect steady-state visual evoked potentials elicited by high frequency stimulation: BCI application," Biomed. Tech. Eng., vol. 55, no. 3, pp. 173-182, Jan. 2010.

[9] W. Nan, C.M. Wong, B. Wang, F. Wan, P.U. Mak, P.I. Mak, and M.I. Vai, "A comparison of minimum energy combination and canonical correlation analysis for SSVEP detection," in EMBS Conference on Neural Engineering, IEEE, pp. 469-472, Apr. 2011.

[10] F. Gembler, P. Stawicki, and I. Volosyak, "Autonomous parameter adjustment for SSVEP-Based BCIs with a novel BCI wizard," Front. Neurosci., vol. 9, p. 474, Dec. 2015.

[11] G. Pfurtscheller, B. Allison, C. Brunner, G. Bauernfeind, T. SolisEscalante, R. Scherer, T. O. Zander, G. Mueller-Putz, C. Neuper, and N. Birbaumer, "The hybrid BCI.," Front. Neurosci., vol. 4, p. 30, Jan. 2010.

[12] S. Amiri, R. Fazel-Rezai, and V. Asadpour, "A Review of Hybrid Brain-computer interface Systems," Advances in HumanComputer Interaction, vol. 2013, pp. 1-8, 2013.

[13] T. Zander, M. Gaertner, C. Kothe, and R. Vilimek, "Combining Eye Gaze Input With a Brain-Computer Interface for Touchless Human-Computer Interaction," International Journal of HumanComputer Interaction, vol. 27, no. 1, pp. 38-51, Dec. 2010.

[14] S. Kishore, M. G. Franco, C. Hintemüller, C. Kapeller, C. Guger, M. Slater, K. J. Blom, "Comparison of SSVEP BCI and Eye Tracking for Controlling a Humanoid Robot in a Social Environment," Presence Teleoperators and Virtual Environments., vol. 23, no. 3, pp. 242-252, Oct. 2014.

[15] A. Évain, F. Argelaguet, G. Casiez, N. Roussel, and A. Lécuyer, "Design and evaluation of fusion approach for combining brain and gaze inputs for target selection," Front. Neurosci., vol. 10, p. 454, Oct. 2016.

[16] P. Stawicki, F. Gembler, A. Rezeika, and I. Volosyak, "A Novel Hybrid Mental Spelling Application Based on Eye Tracking and SSVEP-Based BCI," Brain Sciences, vol. 7, no. 12, p. 35, Apr. 2017.

[17] I. Volosyak, "SSVEP-based Bremen-BCI interface-boosting information transfer rates.," J. Neural Eng., vol. 8, no. 3, p. 036020, Jun. 2011.

[18] M. Mulvenna, G. Lightbody, E. Thomson, P. McCullagh, M. Ware, and S. Martin, "Realistic expectations with brain computer interfaces," Jnl of Assistive Technologies, vol. 6, no. 4, pp. 233244, Nov. 2012.

[19] L. Galway, C. Brennan, P. McCullagh, and G. Lightbody, "BCI and Eye Gaze: Collaboration at the Interface," Lecture Notes in Computer Science, chapter 20, vol. 9183, Springer International Publishing, Cham, pp. 199-210, 2015.

[20]Y. Renard, F. Lotte, G. Gibert, M. Congedo, E. Maby, V. Delannoy, O. Bertrand, and A. Lécuyer, "OpenViBE: An OpenSource Software Platform to Design, Test, and Use BrainComputer Interfaces in Real and Virtual Environments," Presence Teleoperators and Virtual Environments, vol. 19, no. 1, pp. 35-53, Feb. 2010.

[21] O. Friman, I. Volosyak, and A. Graser, "Multiple Channel Detection of Steady-State Visual Evoked Potentials for Braincomputer interfaces," IEEE Trans. Biomed. Eng., vol. 54, no. 4, pp. 742-750, Apr. 2007.

[22] O. Friman, T. Luth, I. Volosyak, and A. Graser, "Spelling with Steady-State Visual Evoked Potentials," in Proceedings of International IEEE EMBS Conference on Neural Engineering, pp. 354-357, 2007.

[23] I. Volosyak, H. Cecotti, D. Valbuena, and A. Graser, "Evaluation of the Bremen SSVEP based BCI in real world conditions," in 2009 IEEE International Conference on Rehabilitation Robotics, IEEE, pp. 322-331, Jun. 2009.

[24] J. R. Wolpaw, H. Ramoser, D. J. McFarland, and G. Pfurtscheller, "EEG-based communication: Improved accuracy by response verification," IEEE Trans. Rehabil. Eng., vol. 6, no. 3, pp. 326333, Sep. 1998.

[25] G. R. Müller-Putz, C. Breitwieser, F. Cincotti, R. Leeb, M. Schreuder, F. Leotta, F., et al (2011). Frontiers: tools for braincomputer interaction: a general concept for a hybrid BCI (HBCI). Front. Neuroinform. 5:30. doi: 10.3389/fninf.2011.00030 